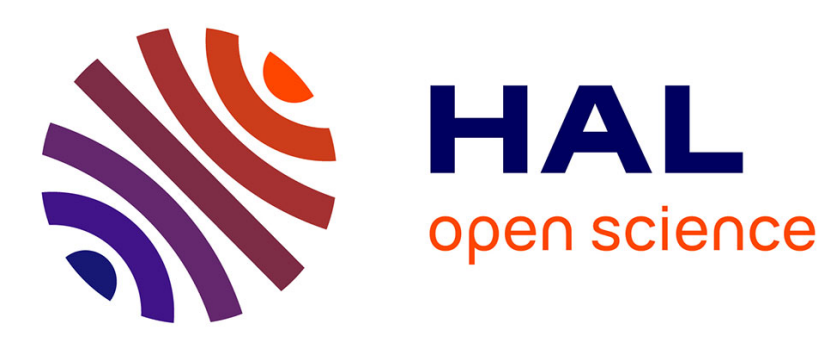

\title{
A coalition formation value for games in partition function form
}

Michel Grabisch, Yukihiko Funaki

\section{To cite this version:}

Michel Grabisch, Yukihiko Funaki. A coalition formation value for games in partition function form.

European Journal of Operational Research, 2012, pp.175-185. halshs-00690696

\section{HAL Id: halshs-00690696 \\ https://shs.hal.science/halshs-00690696}

Submitted on 24 Apr 2012

HAL is a multi-disciplinary open access archive for the deposit and dissemination of scientific research documents, whether they are published or not. The documents may come from teaching and research institutions in France or abroad, or from public or private research centers.
L'archive ouverte pluridisciplinaire HAL, est destinée au dépôt et à la diffusion de documents scientifiques de niveau recherche, publiés ou non, émanant des établissements d'enseignement et de recherche français ou étrangers, des laboratoires publics ou privés. 


\title{
A coalition formation value for games in partition function form
}

\author{
Michel GRABISCH* \\ Paris School of Economics \\ Université de Paris I Panthéon-Sorbonne \\ 106-112 Bd. de l'Hôpital, 75013 Paris, France \\ email michel.grabisch@univ-paris1.fr \\ Yukihiko FUNAKI \\ School of Political Science \& Economics \\ Waseda University, Tokyo, Japan \\ email funaki@waseda.jp
}

\begin{abstract}
The coalition formation problem in an economy with externalities can be adequately modeled by using games in partition function form (PFF games), proposed by Thrall and Lucas. If we suppose that forming the grand coalition generates the largest total surplus, a central question is how to allocate the worth of the grand coalition to each player, i.e., how to find an adequate solution concept, taking into account the whole process of coalition formation. We propose in this paper the original concepts of scenario-value, process-value and coalition formation value, which represent the average contribution of players in a scenario (a particular sequence of coalitions within a given coalition formation process), in a process (a sequence of partitions of the society), and in the whole (all processes being taken into account), respectively. We give also two axiomatizations of our coalition formation value.
\end{abstract}

Keywords: game theory, coalition formation, games in partition function form, Shapley value

\footnotetext{
${ }^{*}$ Corresponding author. Tel (+33) 1-44-07-82-85, Fax (+33) 1-44-07-83-01, email michel.grabisch@univ-paris1.fr
} 


\section{Introduction}

The coalition formation problem is one of the important issues of economics and game theory, both in cooperative and non-cooperative games. There are several attempts to analyze this problem (see, e.g., Ray and Vohra [15], Diamantoudi and Xue [6], Funaki and Yamato [9], and Bloch [3]).

If we suppose that forming the grand coalition generates the largest total surplus, it is natural to assume that the grand coalition structure will eventually form after some negotiations. Then, the worth of the grand coalition has to be allocated to the individual players, according to the contribution of each player. The question is how to do that, taking into account the whole process of coalition formation.

In a coalition formation problem, it is important to consider the structure of the society (i.e., how it is partitioned), since it may affect the worth of a given coalition. This refers in economy to situations with externalities. Such situations are best represented by games in partition function form (PFF games for short; they are also often called games with externalities) introduced by Thrall and Lucas [16] (see also Funaki and Yamato [9]). A partition function assigns a worth to each pair consisting of a coalition and a coalition structure (partition) which contains that coalition. Such pairs are called embedded coalitions.

Then the above problem of allocation of the worth of the grand coalition amounts to defining a suitable solution concept or value for PFF games. For TU games, one of the best known solutions is the Shapley value. This solution concept is based on the marginal contribution of players when they enter the game one by one, considering all possible orders. There are already many attempts to define a modification of the Shapley value for PFF games, e.g., by Myerson [14], Bolger [4], Pham Do and Norde [7], de Clippel and Serrano [5], Albizuri et al. [1], Macho-Stadler et al. [13], etc. They proposed several new kinds of null player or dummy player axioms, and carrier axioms, which are extensions of the original axioms in TU games. Then the resulting formulas are averaging of marginal contributions of players when the players enter the game one by one. However, these approaches do not reflect the process of coalition formation from singletons coalition structure to the grand coalition structure. Since in TU games, the process of entering of the players is expressed by some order on coalitions, in PFF games the process of coalition formation should concern not only coalitions but also the corresponding coalitions structures. Hence, it should be expressed by an order on embedded coalitions.

Mathematically, the set of embedded coalitions has a much more complicated structure than the lattice structure of coalitions in a TU game, however, 
they share similar properties, for example, all maximal chains (paths from the minimal element to the maximal element) are of the same length. This means that a coalition formation process from the singleton coalition structure to the grand coalition structure in a PFF game has always the same number of steps.

Our idea is to take advantage of the structure of embedded coalitions endowed with a suitable ordering, where maximal chains depict the different ways of forming coalitions. Doing so, we can propose a new value for PFF games, which is rooted in the process of coalition formation, and consequently differs from the classical view of the Shapley value.

The rest of the paper is organized as follows. Section 2 introduces embedded coalitions and their structure, Section 3 gives a motivating example showing the insufficiency of the values proposed so far, and Section 4 gives the definition of scenarios and processes. Section 5 introduces our coalition formation value, through scenario-values and process-values. Next, we introduce the axioms giving rise to what we call the egalitarian scenario-value, and we give also a second axiomatization of it. Then we give an explicit expression of the coalition formation value, and finally we give the relation with the Shapley value when the game has no externalities. Section 6 applies the coalition formation value to the motivating example, and Section 7 concludes the paper by giving a comparison with other values for PFF games. Long proofs are relegated to the Appendix.

\section{Partitions and embedded coalitions}

Let $N:=\{1,2, \ldots, n\}$ be the set of players. We denote by $S, T, \ldots$, the subsets of $N$, called coalitions, and their cardinality by $s, t, \ldots$ A partition $\pi:=\left\{S_{1}, \ldots, S_{k}\right\}$, also called coalition structure, is a collection of disjoint nonempty coalitions $S_{1}, \ldots, S_{k}$ with $\cup_{l=1}^{k} S_{l}=N$. Subsets $S_{1}, \ldots, S_{k}$ are called blocks of $\pi$. We denote by $\Pi(N)$ or $\Pi(n)$ the set of all possible partitions of $N$. A natural ordering of partitions is given by the notion of "coarsening" or "refinement". Taking $\pi, \pi^{\prime}$ partitions in $\Pi(N)$, we say that $\pi$ is a refinement of $\pi^{\prime}$ or $\pi^{\prime}$ is a coarsening of $\pi$, denoted by $\pi \leq \pi^{\prime}$, if any block of $\pi$ is contained in a block of $\pi^{\prime}$ (or equivalently, every block of $\pi^{\prime}$ fully decomposes into blocks of $\pi)$. Then $(\Pi(N), \leq)$ is a lattice, called the partition lattice. With this ordering, the bottom element of the lattice is the finest partition $\pi^{\perp}:=\{\{1\}, \ldots,\{n\}\}$ called the singletons coalition structure, while the top element is the coarsest partition $\pi^{\top}:=\{N\}$ called the grand coalition structure.

An embedded coalition is a pair $(S, \pi)$, where $S \in 2^{N} \backslash\{\emptyset\}$ is a coalition, 
and $\pi$ is a coalition structure such that $S \in \pi$. We also call $S$ the base coalition of $(S, \pi)$. We denote by $\Sigma(N)$ (or by $\Sigma(n))$ the set of embedded coalitions on $N$. For the sake of concision, we often denote by $S \pi$ the embedded coalition $(S, \pi)$, and omit braces and commas for subsets (example: $12\{12,3\}$ instead of $(\{1,2\},\{\{1,2\},\{3\}\}))$. We propose the following order relation on embedded coalitions:

$$
(S, \pi) \sqsubseteq\left(S^{\prime}, \pi^{\prime}\right) \Leftrightarrow S \subseteq S^{\prime} \text { and } \pi \leq \pi^{\prime}
$$

(this can be read: $(S, \pi)$ is before $\left.\left(S^{\prime}, \pi^{\prime}\right)\right)$ Evidently, the top element of this ordered set is $\left(N, \pi^{\top}\right)$ (denoted more simply by $N\{N\}$ according to our convention). However, due to the fact that the empty set is not allowed in $(S, \pi)$, there is no bottom element in the ordered structure $(\Sigma(N)$, $)$. Indeed, all elements of the form $\left(\{i\}, \pi^{\perp}\right)$ are minimal elements (i.e., there is no smaller element than them). For mathematical convenience, we introduce an artificial bottom element $\perp$ to $\Sigma(N)$ (it could be considered as $\left(\emptyset, \pi^{\perp}\right)$ ), and denote $\Sigma(N)_{\perp}:=\Sigma(N) \cup\{\perp\}$. We give as illustration the partially ordered set $\left(\Sigma(N)_{\perp}\right.$, $)$ with $n=3$ (Fig. 1).

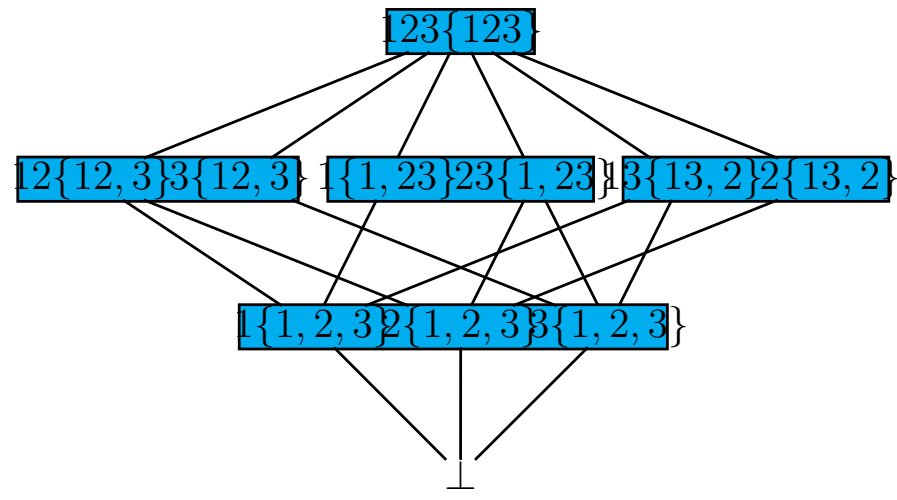

Figure 1: Diagram of $\left(\Sigma(N)_{\perp}\right.$, $)$ for $n=3$. Elements with the same partition are framed in grey.

Definition 1. A game in partition function form (PFF-game in short) on $N$ is a mapping $v: \Sigma(N)_{\perp} \rightarrow \mathbb{R}$, such that $v(\perp)=0$. The set of all PFF-games on $N$ is denoted by $\Gamma(N)$.

Following the usual interpretation, the payoff for coalition $S$ depends on the situation of the outsiders, depicted by the partition $\pi$, which represents the externalities in an economy. 
To be meaningful, we assume that forming the grand coalition generates the largest total surplus, i.e., $v(N\{N\}) \geq \sum_{S \in \pi} v(S, \pi)$, for all $\pi \in \Pi(N)$. Hence, we consider economic environments where doing so is the best for the society and the total surplus $v(N\{N\})$ is distributed among the players in the society.

We recall that in a partially ordered set $(P, \leq)$ with a bottom element $\perp$ and a top element $T$, a chain from $\perp$ to $T$ is a totally ordered sequence of elements of $P$ including $\perp, \top$. The chain is maximal if no other chain can contain it (equivalently, if between two consecutive elements $x_{i}, x_{i+1}$ of the sequence, there is no element $x \in P$ such that $\left.x_{i}<x<x_{i+1}\right)$. If no ambiguity occurs, we say maximal chain instead of maximal chain from $\perp$ to $T$. The set of maximal chains in $P$ is denoted by $\mathcal{C}(P)$. The length of a maximal chain is the number of elements of the sequence minus 1 . If all maximal chains have the same length, this length is the height of the partially ordered set. In Fig. 1 , the sequence $\perp, 1\{1,2,3\}, 1\{1,23\}, 123\{123\}$ is a maximal chain, and there are 9 maximal chains in total, all of length 3 , hence the height of $\left(\Sigma(123)_{\perp}, \sqsubseteq\right)$ is 3. In [12], it is proved that $\left(\Sigma(N)_{\perp}, \sqsubseteq\right)$ is a lattice, whose maximal chains have all the same length $n$. The combinatorial complexity of $\left(\Sigma(N)_{\perp}, \sqsubseteq\right)$ is far beyond the complexity of the Boolean lattice of coalitions in a TU game. In [12], it is proved that the number of maximal chains from $\perp$ to $(N,\{N\})$ is $\left|\mathcal{C}\left(\Sigma(n)_{\perp}\right)\right|=\frac{(n !)^{2}}{2^{n-1}}$. For simplicity, we put $c:=\left|\mathcal{C}\left(\Sigma(n)_{\perp}\right)\right|$ and it is shown in $[12]$ that $|\mathcal{C}(\Pi(n))|=\frac{c}{n}$.

\section{A motivating example}

We consider Cournot oligopoly games where players are firms, particularly the simple case where the games are symmetric, in the sense that the worth of the embedded coalition depends only on the number of blocks of the partition, i.e., $v(S, \pi)=v_{k}$, where $\pi=\left\{S, S_{1}, \ldots, S_{k-1}\right\}$ (see below, and Funaki and Yamato for practical examples of such games [9]).

Because most of the values for PFF games presented so far obey the classical symmetry axiom, saying that the value is invariant under permutations, (see Section 7 for details), they will lead to the equal division of $v(N,\{N\})$, for each firm. However, this result is too simplistic and does not reflect in which way the firms merged together to form the grand coalition. Let us consider two extreme cases of coalition formation. In the first one, a new firm joins the current coalition at each step, i.e., starting from firm 1 alone, firm 2 merges with firm 1, then firm 3 joins, etc. This leads to the sequence of partitions $\{1,2,3,4, \ldots, n\},\{12,3,4, \ldots, n\},\{123,4, \ldots, n\}, \ldots,\{N\}$. Note that this way of forming the grand coalition is exactly what Shapley de- 
scribed when defining his value for TU-games. By contrast to this very unbalanced way of forming the grand coalition, the second case considers that first firms merge by pairs, then these pairs merge again by pair, and so on till forming the grand coalition. This leads to a perfectly balanced way of forming $N$, provided $n$ is of the form $2^{k}$. For example, with $n=8$, we get: $\{1,2,3,4,5,6,7,8\}$ then $\{12,34,56,78\}$, then $\{1234,5678\}$ and $\{N\}$. It is hard to admit that in both situations, the contribution of a firm $i$ should be the same, i.e., $v(N,\{N\}) / n$.

Let us be more specific and consider the following Cournot model with $n$ identical firms. Let $x_{i}$ be firm $i$ 's output $(i=1, \ldots, n)$. The inverse demand function is given by $p=a-\sum_{i=1}^{n} x_{i}$, and the total cost function of firm $i$ is $c x_{i}$, where $a>c>0$. Given a coalition structure $\pi=\left\{S_{1}, S_{2}, \ldots, S_{k}\right\}$, we assume that each coalition $S_{j}$ is a player who chooses the total output level of its firms to maximize the sum of their profits, given the output levels of other coalitions. Then it is easy to check that the total profit of each coalition $S_{j}$ at a unique Nash equilibrium is given by $\frac{(a-c)^{2}}{(k+1)^{2}}$. Without loss of generality, we assume that $a-c=1$. This gives

$$
v_{k}=\frac{1}{(k+1)^{2}}
$$

This implies $v_{n-1}-v_{n}<v_{n-2}-v_{n-1}<\ldots<v_{2}-v_{3}<v_{1}-v_{2}$. Consider now the first way (Shapley-like) of forming the grand coalition: $\{1,2,3,4, \ldots, n\}$, $\{12,3,4, \ldots, n\},\{123,4, \ldots, n\}, \ldots,\{N\}$. The marginal contribution of firm 2 is proportional to $v_{n-1}-v_{n}$, much smaller than $v_{1}-v_{2}$, which is the contribution of firm $n$, entering the last. Therefore, it is counterintuitive that their values are equal.

On the other hand, the way the grand coalition is formed in the second situation is perfectly symmetric, since any permutation on the firms does not change the role of the firms in the coalition formation (which is not true in the previous situation: permuting firms 1 and $n$ makes them have a totally different role): Every single firm makes a two-person coalition when making the first coalition. Next, two-person coalitions merge into 4-person coalitions, and so on. In each step, both coalitions have the same power. Then one expects that every firm has an equal contribution.

In summary, we believe that the exact way how coalitions form (we call this a process, see Definition 2) should be taken into account into the computation of the value. So far, the values presented in the literature do not possess this feature. 


\section{Processes and scenarios}

Let us describe what we mean by a process of coalition formation. We consider that we start from the society of individual players, and after several steps of negotiation, the grand coalition has formed. This is guaranteed by our assumption above. Moreover, we consider that at each step, exactly two blocks (coalitions) of the current partition merge to form a new coalition. Also, we discard the possibility for a block to split in several blocks, for this would considerably complicate the analysis, and is anyway unlikely to occur in economic environments where the grand coalition generates the largest surplus. In summary:

Definition 2. A coalition formation process $\mathbf{p}$ is any maximal chain in $\Pi(n)$, i.e., a sequence of partitions starting from $\pi^{\perp}$ (singleton coalition structure) and ending at $\{N\}$ (the grand coalition). The set of processes is therefore $\mathcal{C}(\Pi(n))$, which we denote simply by $\mathcal{P}$.

Our second fundamental ingredient is the notion of scenario. Let us consider some process p. A process is an "external" description of how coalitions form, as it could be seen by some observer outside the game. Let us now consider an "internal" view by some player $i \in N$. At the initial step, player $i$ is alone, and during the subsequent steps, $\mathrm{s} /$ he will join with some other blocks, to become finally included in $N$. Hence, the sequence of embedded coalitions $\left(S_{1}, \pi_{1}\right), \ldots,\left(S_{n}, \pi_{n}\right)$, where the sequence $\pi_{1}, \ldots, \pi_{n}$ is p itself, and $S_{1}=\{i\}, S_{n}=N$ and all $S_{2}, \ldots, S_{n-1}$ contain $i$, is the internal view of the process $\mathbf{p}$, seen by player $i$. We call this the scenario for player $i$ (in the process $\mathbf{p}$ ).

Definition 3. Let $\mathbf{p}=\pi_{1}, \ldots, \pi_{n-1}, N$ be a process. A scenario $\mathbf{s}$ induced by process $\mathbf{p}$ is any maximal chain in $\Sigma(n)_{\perp}$ of the form $\perp, S_{1} \pi_{1}, \ldots, S_{n-1} \pi_{n-1}, N\{N\}$. We denote by $\mathbf{s} \leftarrow \mathbf{p}$ the fact that $\mathbf{s}$ is induced by $\mathbf{p}$.

The set of all scenarios, considering all processes, is therefore $\mathcal{C}\left(\Sigma(n)_{\perp}\right)$, denoted by $\mathcal{S}$ for simplicity.

For a given process $\mathbf{p}$, there are $n$ scenarios $\mathbf{s}_{i}, i \in N$. For example, in a 3 persons game with $N=\{1,2,3\}$, we have three processes:

$$
\begin{aligned}
& \mathbf{p}_{1}:\{1,2,3\} \rightarrow\{12,3\} \rightarrow\{123\} \\
& \mathbf{p}_{2}:\{1,2,3\} \rightarrow\{13,2\} \rightarrow\{123\} \\
& \mathbf{p}_{3}:\{1,2,3\} \rightarrow\{1,23\} \rightarrow\{123\}
\end{aligned}
$$

In process $\mathbf{p}_{2}$, first players 1 and 3 merge, then coalitions 13 and 2 merge and form the grand coalition. A process describes how exactly the coalition structure evolves step by step, by a process of coalition formation. 
Each coalition process has three scenarios. In the above example, process $\mathbf{p}_{2}$ has the following three scenarios:

$$
\begin{aligned}
& \mathbf{s}_{1}: \perp \rightarrow 1\{1,2,3\} \rightarrow 13\{13,2\} \rightarrow 123\{123\} \\
& \mathbf{s}_{2}: \perp \rightarrow 2\{1,2,3\} \rightarrow 2\{13,2\} \rightarrow 123\{123\} \\
& \mathbf{s}_{3}: \perp \rightarrow 3\{1,2,3\} \rightarrow 13\{13,2\} \rightarrow 123\{123\}
\end{aligned}
$$

In $\mathbf{s}_{1}$, player 1 first merges with player 3 at the singletons coalition structure, then this base coalition 13 containing player 1 merges with player 2 and becomes the grand coalition structure.

In a scenario s, some elements play a special role. We consider those elements $S \pi$ such that in the sequence of elements of $\mathbf{s}$ from bottom to top, $S \pi$ is the last element with base coalition $S$. They are called terminal elements. Specifically, let us denote $\mathbf{s}$ by

$\mathbf{s}=\left\{\perp, S_{1} \pi_{1,1}, \ldots, S_{1} \pi_{1, m_{1}}, S_{2} \pi_{2,1}, \ldots, S_{2} \pi_{2, m_{2}}, \ldots, S_{k} \pi_{k, 1}, \ldots, S_{k} \pi_{k, m_{k}}, N\{N\}\right\}$,

with $S_{1} \subsetneq \cdots \subsetneq S_{k} \neq N$. Then the terminal elements are $S_{i} \pi_{i, m_{i}}, i=$ $1, \ldots, k$. We denote by $\mathcal{F}(\mathbf{s})$ this family of elements. A terminal element is an embedded coalition such that the base coalition of the embedded coalition changes at the next step in the scenario.

Example 1. We consider 4 players and the following process $\mathbf{p}$ :

$$
\{1,2,3,4\} \rightarrow\{13,2,4\} \rightarrow\{13,24\} \rightarrow\{1234\} .
$$

and the four different scenarios in $\mathbf{p}$ where terminal elements are in bold:

$$
\begin{aligned}
& \mathbf{s}_{1}: \perp \rightarrow \mathbf{1}\{\mathbf{1}, \mathbf{2}, \mathbf{3}, \mathbf{4}\} \rightarrow 13\{13,2,4\} \rightarrow \mathbf{1 3}\{\mathbf{1 3}, \mathbf{2 4}\} \rightarrow \mathbf{N}\{\mathbf{N}\} \\
& \mathbf{s}_{2}: \perp \rightarrow 2\{1,2,3,4\} \rightarrow \mathbf{2}\{\mathbf{1 3}, \mathbf{2}, \mathbf{4}\} \rightarrow \mathbf{2 4}\{\mathbf{1 3}, \mathbf{2 4}\} \rightarrow \mathbf{N}\{\mathbf{N}\} \\
& \mathbf{s}_{3}: \perp \rightarrow \mathbf{3}\{\mathbf{1}, \mathbf{2}, \mathbf{3}, \mathbf{4}\} \rightarrow 13\{13,2,4\} \rightarrow \mathbf{1 3}\{\mathbf{1 3}, \mathbf{2 4}\} \rightarrow \mathbf{N}\{\mathbf{N}\} \\
& \mathbf{s}_{4}: \perp \rightarrow 4\{1,2,3,4\} \rightarrow \mathbf{4}\{\mathbf{1 3}, \mathbf{2}, \mathbf{4}\} \rightarrow \mathbf{2 4}\{\mathbf{1 3}, \mathbf{2 4}\} \rightarrow \mathbf{N}\{\mathbf{N}\}
\end{aligned}
$$

In scenario $\mathbf{s}_{1}$, player 1 first merges with player 3 , then this coalition remains unchanged in the next step, and finally base coalition 13 merges with coalition 24, and becomes the grand coalition. Note that the second embedded coalition is not a terminal element because at the next step the base coalition does not change (player 1 is not concerned by the move).

\section{$5 \quad$ Values for coalition formation processes}

In this section, we introduce our concept of value for PFF games. Since it is based on an interpretation in terms of coalition formation, we call it 
a coalition formation value. This name emphasizes that it differs from the usual coalitional values, like the Shapley value and its generalizations to PFF games. Their exact relationship will be studied in Section 5.5.

\subsection{Scenario-values, process-values and values}

Consider again the initial state of the society where all players are individual, and no coalition has formed. We know from the previous section that under our assumptions finally the grand coalition will form, following some process $\mathbf{p}$, and our aim is to share the total surplus of the game among the players, taking into account the contribution of each player during the coalition formation process. Here, two situations can arise: either we know precisely the process $\mathbf{p}$ (i.e., the grand coalition has formed and we observed how it was formed, like in the Cournot example in Section 3), or we do not know it, either because we were not able to observe the process, or because the process has not yet realized. In the first situation, it seems natural to make the calculation of the sharing by using only those embedded coalitions $(S, \pi)$ which are related to the process $\mathbf{p}$, in other words, all $(S, \pi)$ in the $n$ scenarios of $\mathbf{p}$, the other ones being irrelevant. We call the sharing obtained in this way a process-value. In the second situation, our ignorance obliges us to use a priori all embedded coalitions, since any process may realize or have realized. If we have no knowledge at all about the process, the principle of insufficient reason tells us that we should consider equally all processes; therefore, our value will be the arithmetic mean of the process-values, for all possible processes $\mathbf{p} \in \mathcal{P}$. Otherwise, we may define some weight vector on each process (or even on scenarios), representing, e.g., a probability of occurence of each process, and compute a weighted arithmetic mean. Hence, depending on the practical situation under consideration, either the process-value or the (weighted) value is better suited.

Let us now consider a given process $\mathbf{p}$ and define the process-value. Unlike global games [11] which assigns worths to partitions - and therefore processes fully make sense for them, PFF games assign worths to coalitions given a partition, and therefore we must consider the $n$ scenarios induced by a process, i.e., all possible sequences of embedded coalitions pertaining to the same process. In each scenario, we should compute the contribution of each player, which we call the scenario-value.

Summarizing the above discussion, we are lead to the following definition.

Definition 4. (i) A scenario-value is a mapping $\psi: \Gamma(N) \rightarrow \mathbb{R}^{n \times|\mathcal{S}|}$. Components of $\psi(v)$ are denoted by $\psi_{i}^{\mathbf{s}}(v)$ for scenario $\mathbf{s}$ and player $i$. 
If there is no confusion, we also call each component $\psi_{i}^{\mathbf{s}}(v)$ a scenariovalue.

(ii) A process-value is a mapping $\psi: \Gamma(N) \rightarrow \mathbb{R}^{n \times|\mathcal{P}|}$. Components of $\psi(v)$ are denoted by $\psi_{i}^{\mathbf{p}}(v)$ for process $\mathbf{p}$ and player $i$. If there is no confusion, we also call each component $\psi_{i}^{\mathbf{p}}(v)$ a process-value. Any scenario-value $\psi$ induces a process-value (denoted with some abuse by the same letter $\psi$ ) by:

$$
\psi_{i}^{\mathbf{p}}(v):=\frac{1}{n} \sum_{\mathbf{s} \leftarrow \mathbf{p}} \psi_{i}^{\mathbf{s}}(v), \quad i \in N, \mathbf{p} \in \mathcal{P} .
$$

(iii) A value is a mapping $\psi: \Gamma(N) \rightarrow \mathbb{R}^{n}$. Components of $\psi(v)$ are denoted by $\psi_{i}(v)$ for player $i$. Any scenario-value or process-value $\psi$ induces a value by:

$$
\psi_{i}(v):=\frac{n}{c} \sum_{\mathbf{p} \in \mathcal{P}} \psi_{i}^{\mathbf{p}}(v)=\frac{1}{c} \sum_{\mathbf{s} \in \mathcal{S}} \psi_{i}^{\mathbf{s}}(v),
$$

where we recall that $c$ is the number of scenarios (maximal chains in $\Sigma(N))$. More generally, weighted values can be considered as well: $\psi_{i}(v)=\sum_{\mathbf{p} \in \mathcal{P}} w_{\mathbf{p}} \psi_{i}^{\mathbf{p}}(v)$, with $w_{\mathbf{p}} \geq 0$ and $\sum_{\mathbf{p} \in \mathcal{P}} w_{\mathbf{p}}=1$, or $\psi_{i}(v)=$ $\sum_{\mathbf{s} \in \mathcal{S}} w_{\mathbf{s}} \psi_{i}^{\mathbf{s}}(v)$.

\subsection{Axioms}

A scenario-value satisfies linearity $(\mathbf{L})$ if it is linear on the set of PFF-games. We define similarly linearity for process-values and values.

Proposition 1. If $\psi$ is a linear scenario-value, then for all $v \in \Gamma(N)$

$$
\psi_{i}^{\mathbf{s}}(v)=\sum_{S \pi \in \Sigma(n)} \gamma_{\mathbf{s}, S \pi}^{i} v(S \pi), \quad \forall i \in N, \forall \mathbf{s} \in \mathcal{S}
$$

for some real constants $\gamma_{\mathbf{s}, S \pi}^{i}$ (and similarly for a linear process-value, with constants $\gamma_{\mathbf{p}, S \pi}^{i}$, and for a linear value, with constants $\left.\gamma_{S \pi}^{i}\right)$.

Proof. As usual, consider the elementary PFF-games $e_{S \pi}\left(S^{\prime} \pi^{\prime}\right):=1$ iff $S \pi=$ $S^{\prime} \pi^{\prime}$ and 0 otherwise, for all $S \pi \in \Sigma(n)$. Then linearity for scenario-values implies:

$$
\psi(v)=\psi\left(\sum_{S \pi \in \Sigma(n)} v(S \pi) e_{S \pi}\right)=\sum_{S \pi \in \Sigma(n)} v(S \pi) \psi\left(e_{S \pi}\right),
$$

hence the result, letting $\left.\psi_{i}^{\mathbf{s}}\left(e_{S \pi}\right)\right)=: \gamma_{\mathbf{s}, S \pi}^{i}$ for $i \in N, \mathbf{s} \in \mathcal{S}$. 
Definition 5. Let us consider $i \in N$, a scenario s, and denote by $S^{-} \pi^{-}$ the last element in $\mathbf{s}$ not containing $i$, and $S \pi$ its successor in $\mathcal{F}(\mathbf{s})$, as in Figure 2. Player $i$ is null in scenario $\mathbf{s}$ for $v$ if $v(S \pi)=v\left(S^{-} \pi^{-}\right)$. Player $i$ is null for $v$ if $i$ is null for every scenario $\mathbf{s}$.

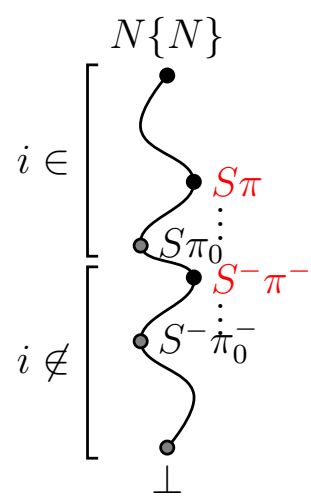

Figure 2: Notation used for scenario s (terminal elements are in black, others are in grey)

Scenario Null axiom (SN): If $i$ is null in scenario $\mathbf{s}$ for $v$, then $\psi_{i}^{\mathbf{s}}(v)=0$.

If $i$ is null for every scenario $\mathbf{s}$, then $\psi_{i}(v)=0$ (null axiom for the induced value), but the converse does not hold, that is, if $\psi_{i}(v)=0$ holds, then $i$ is not necessary null in every scenario.

Proposition 2. Under (L) and (SN), for every scenario s, every player $i$, the scenario-value reads

$$
\psi_{i}^{\mathbf{S}}(v)=\gamma_{\mathbf{s}, S \pi}^{i}\left(v(S \pi)-v\left(S^{-} \pi^{-}\right)\right)
$$

with notation of Fig 2, and with real constants $\gamma_{\mathbf{s}, S \pi}^{i}, i \in N, \mathbf{s} \in \mathcal{S}$.

Proof. Take any scenario $\mathbf{s}$, and define $v(S \pi)=v\left(S^{-} \pi^{-}\right)=1$ and $v\left(S^{\prime} \pi^{\prime}\right)=0$ otherwise. Then $i$ is null for $v$ in $\mathbf{s}$. Then by (SN) we get $\gamma_{\mathbf{s}, S^{-} \pi^{-}}^{i}+\gamma_{\mathbf{s}, S \pi}^{i}=0$. Now take $v^{\prime}=v$, except on a single element $S^{\prime} \pi^{\prime} \in \Sigma(n)$ different from $S^{-} \pi^{-}, S \pi$. Since $i$ is still null for $v^{\prime}$ in $\mathbf{s}$, we get by (SN) that $\gamma_{\mathbf{s}, S^{\prime} \pi^{\prime}}^{i}=0$.

The following is the usual symmetry axiom, but unlike in the classical case, its effect on the simplification of the gamma coefficients will be much weaker. 
Symmetry axiom for the scenario-value (SS): For any $i \in$ $N$, any $\mathbf{s} \in \mathcal{S}$, and any permutation $\sigma$ on $N$, it holds

$$
\psi_{i}^{\mathbf{s}}(v)=\psi_{\sigma(i)}^{\sigma(\mathbf{s})}\left(v \circ \sigma^{-1}\right)
$$

with $\sigma(\mathbf{s}), \sigma(S, \pi)$ defined naturally as follows: $\sigma(S, \pi)=(\sigma(S), \sigma(\pi))$, where $\sigma(S)=\{\sigma(i) \mid i \in S\}, \sigma(\pi)=\{\sigma(S) \mid S \in \pi\}$, and $\sigma(\mathbf{s})=\{\sigma(S, \pi) \mid(S, \pi) \in \mathbf{s}\}$.

For any scenario $\mathbf{s}:=\left(\perp, S_{1} \pi_{1}, \ldots, S_{n} \pi_{n}=N\{N\}\right)$, we can define what we call its signature, which is the part of the scenario being invariant under permutations. For this, we need an unambiguous way to arrange blocks in the embedded coalitions (i.e., the components of the partitions) of s. First, for $\pi_{1}=\pi^{\perp}$, an order on the blocks (singletons) is fixed. Then, for any $\pi_{k}$, $k=2, \ldots, n-1$, the blocks are arranged in decreasing order of their size, and blocks of same size are arranged in the lexicographic order induced by the order on singletons. We call this the induced ordering. Now, the signature of $\mathbf{s}$, assuming that blocks are arranged with the induced ordering, is the sequence $\tau(\mathbf{s}):=\left(\left(s_{1}, \rho\left(\pi_{1}\right),\left(i_{1}, j_{1}\right)\right), \ldots,\left(s_{n-1}, \rho\left(\pi_{n-1}\right),\left(i_{n-1}, j_{n-1}\right)\right),(n,(n))\right)$, where $\rho\left(\pi_{k}\right)$ is the sequence of cardinalities of blocks of $\pi_{k}$, and $\left(i_{k}, j_{k}\right)$ denotes the index numbers of blocks in $\pi_{k}$ which are merged to form $\pi_{k+1}{ }^{1}$. Note that there are at most $n$ ! different scenarios with the same signature, generated by all permutations on $N$.

Example 2. Consider the scenario s given by

$$
1\{1,2,3,4,5\} \rightarrow 12\{12,3,4,5\} \rightarrow 12\{12,34,5\} \rightarrow 125\{125,34\} \rightarrow 12345\{12345\} .
$$

Its signature's long and shortest versions are

$$
\begin{aligned}
\tau(\mathbf{s})= & ((1,(1,1,1,1,1),(1,2)),(2,(2,1,1,1),(2,3)),(2,(2,2,1),(1,3)), \\
& (3,(3,2),(1,2)),(5,(5))) \\
\tau(\mathbf{s})= & (1 ;(1,2),(2,3),(1,3)) .
\end{aligned}
$$

The scenario $\mathbf{s}^{\prime}$ given by

$4\{1,2,3,4,5\} \rightarrow 14\{14,2,3,5\} \rightarrow 14\{14,2,35\} \rightarrow 124\{124,35\} \rightarrow 12345\{12345\}$

has the same signature and is obtained from $\mathbf{s}$ by the permutation 41532 .

\footnotetext{
${ }^{1}$ This is the long version of the signature, which contains many redundant information. An equivalent shortest (and therefore irredundant) version is $\tau(\mathbf{s})=\left(i ;\left(i_{1}, j_{1}\right),\left(i_{2}, j_{2}\right), \ldots\right.$, $\left(i_{n-2}, j_{n-2}\right)$ ), where $i$ is the index of $S_{1}$ in $\pi_{1}$.
} 
Proposition 3. Under (L), (SN) and (SS), the scenario-value takes the form

$$
\psi_{i}^{\mathbf{s}}(v)=\gamma_{\tau(\mathbf{s}),|\pi|}^{i}\left(v(S \pi)-v\left(S^{-} \pi^{-}\right)\right),
$$

for any scenario $\mathbf{s}$ and player $i$, with same notation as in Figure 2, $\tau(\mathbf{s})$ is the signature of the scenario, and $|\pi|$ is the number of blocks of $\pi$.

Proof. We know from (L) and (SN) that $\psi_{i}^{\mathbf{s}}(v)$ takes the form (3). Hence, for any permutation $\sigma$ on $N$ we have for any game $v$

$$
\psi_{\sigma(i)}^{\sigma(\mathbf{s})}\left(v \circ \sigma^{-1}\right)=\gamma_{\sigma(\mathbf{s}), \sigma(S \pi)}^{\sigma(i)}\left(v(S \pi)-v\left(S^{-} \pi^{-}\right)\right) .
$$

The (SS) axiom entails $\gamma_{\mathbf{s}, S \pi}^{i}=\gamma_{\sigma(\mathbf{s}), \sigma(S, \pi)}^{\sigma(i)}$, and this holds for any permutation $\sigma$.

Considering the coefficient $\gamma_{\mathbf{s}, S \pi}^{i}$, we note that $S \pi \in \mathbf{s}$ and $i \in S \backslash S^{-}$. Taking a permutation $\sigma$, it is plain that $\tau(\sigma(\mathbf{s}))=\tau(\mathbf{s}),|\sigma(\pi)|=|\pi|$, and that $\sigma(S \pi) \in \sigma(\mathbf{s}), \sigma(i) \in \sigma(S) \backslash \sigma\left(S^{-}\right)$. Conversely, take any scenario $\mathbf{s}^{\prime}$ of same signature, $S^{\prime} \pi^{\prime} \in \mathbf{s}^{\prime}$ such that $\left|\pi^{\prime}\right|=|\pi|$ and $i^{\prime} \in S^{\prime} \backslash S^{\prime-}$, where $S^{\prime-}$ precedes $S^{\prime}$ in $\mathbf{s}^{\prime}$. Then there exists some permutation $\sigma$ such that $\sigma(\mathbf{s})=\mathbf{s}^{\prime}$ and since $\left|\pi^{\prime}\right|=|\pi|$, we have $\sigma(S \pi)=S^{\prime} \pi^{\prime}$. Now observe that it is not necessarily true that $i^{\prime}=\sigma(i)$, as shown by the following example:

$$
\begin{aligned}
\mathbf{s} & =3\{1,2,3,4,5\} \rightarrow 3\{12,3,4,5\} \rightarrow 34\{12,34,5\} \rightarrow 34\{125,34\} \rightarrow N\{N\} \\
\mathbf{s}^{\prime} & =3\{5,2,3,4,1\} \rightarrow 3\{25,3,4,1\} \rightarrow 34\{25,34,1\} \rightarrow 34\{125,34\} \rightarrow N\{N\},
\end{aligned}
$$

and consider $S \pi=S^{\prime} \pi^{\prime}=N\{N\}$, and $i=i^{\prime}=1$. Then $\sigma$ must necessarily change 1 into 5 , i.e., $\sigma(i) \neq i^{\prime}$. Therefore, the superindex $i$ is necessary.

By analogy with coalitional cooperative games, the unanimity game $u_{S \pi}$ centered on $S \pi$ is defined by $u_{S \pi}\left(S^{\prime} \pi^{\prime}\right)=1$ if $S^{\prime} \pi^{\prime} \sqsupset S \pi$ and 0 otherwise. The last axiom expresses the fact that for the unanimity game $u_{S \pi}$ and any scenario where $S \pi$ is a terminal element, there is equal contribution for all players entering $S$ in the scenario.

Egalitarian axiom (EG): consider a scenario s and $S \pi, S^{-} \pi^{-} \in$ $\mathcal{F}(\mathbf{s})$ where $S^{-} \pi^{-}$is the predecessor of $S \pi$ in $\mathcal{F}(\mathbf{s})$, and $\left|S \backslash S^{-}\right|>$ 1. Then $\forall i, j \in\left|S \backslash S^{-}\right|, \psi_{i}^{\mathbf{s}}\left(u_{S \pi}\right)=\psi_{j}^{\mathbf{s}}\left(u_{S \pi}\right)$.

Proposition 4. Under (L), (SN), (SS) and (EG), the scenario-value takes the form

$$
\psi_{i}^{\mathbf{s}}(v)=\gamma_{\tau(\mathbf{s}),|\pi|}\left(v(S \pi)-v\left(S^{-} \pi^{-}\right)\right)
$$

for any scenario s and player $i \in N$ (same notation as in Figure 2). 
Proof is immediate from (4).

Our last axiom is efficiency.

Scenario-efficiency axiom (SE): for every $\mathbf{s} \in \mathcal{S}, \sum_{i \in N} \psi_{i}^{\mathbf{s}}(v)=$ $v(N\{N\})$. Similarly, we define process-efficiency $(\mathbf{P E})$ as $\sum_{i \in N} \psi_{i}^{\mathbf{p}}(v)=$ $v(N\{N\})$ for all $\mathbf{p} \in \mathcal{P}$, and efficiency (E) as $\sum_{i \in N} \psi_{i}(v)=$ $v(N\{N\})$.

With some abuse, we say that a scenario-value is (process-)efficient if its induced (process-)value is. Obviously, scenario-efficiency implies processefficiency anf efficiency.

Theorem 1. The unique scenario-value satisfying (L), (SN), (SS), (EG) and (SE) is given by

$$
\phi_{i}^{\mathbf{s}}(v)=\frac{1}{\left|S \backslash S^{-}\right|}\left(v(S \pi)-v\left(S^{-} \pi^{-}\right)\right),
$$

with notation of Figure 2. We call it the egalitarian scenario-value.

(see proof in the Appendix) We call coalition formation value the value induced by the egalitarian scenario-value.

\subsection{A second axiomatization}

The egalitarian scenario-value is fully characterized by these axioms, which are very similar to the classical axioms of the Shapley value for TU games. However, every axiom is restricted to a single scenario in a coalition formation process, therefore in some sense we have a local axiomatization. Imposing that each scenario must satisfy these axioms may appear as a strong condition. Our next axiomatization avoids this by using efficiency, the weaker and most global form of efficiency, instead of the strong and local scenarioefficiency.

Consider the unanimity game $u_{S \pi_{0}}$, where $S$ is any nonempty coalition, and $\pi_{0}$ is the finest partition $\pi_{0}$ containing $S$, i.e., $\pi_{0}=\left\{S, \pi_{N \backslash S}^{\perp}\right\}$, where $\pi_{N \backslash S}^{\perp}$ is the partition of $N \backslash S$ in singletons. Then, any embedded coalition with base coalition $S$ has worth 1, and any embedded coalition with base coalition $S^{-} \subset S$ has worth 0 , whatever the externalities are. In this situation, for a fixed $S^{-} \subset S$, it is natural to say that the contribution of a player $i \in$ $S \backslash S^{-}$does not depend on the scenario s, provided that in the scenario $S^{-}$ precedes $S$ (more precisely, $S^{-} \pi^{-}$precedes $S \pi$ in $\mathcal{F}(\mathbf{s})$, for some $\pi, \pi^{-}$). Put more bluntly, the organisation of the players in $N \backslash S$ is irrelevant to the contribution of $i$ for the game $u_{S \pi_{0}}$. 
Independence of irrelevant players axiom (IIP): Consider a nonempty coalition $S \subseteq N$, and $\pi_{0}$ the finest partition containing $S$. Then for every scenarios $\mathbf{s}, \mathbf{s}^{\prime}$ such that $S \pi \in \mathcal{F}(\mathbf{s}), S \pi^{\prime} \in$ $\mathcal{F}\left(\mathbf{s}^{\prime}\right)$, where $\pi, \pi^{\prime}$ are arbitrary partitions containing $S$, it holds

$$
\psi_{i}^{\mathbf{s}}\left(u_{S \pi_{0}}\right)=\psi_{i}^{\mathbf{s}^{\prime}}\left(u_{S \pi_{0}}\right)
$$

for every $i \in S \backslash S^{-}$, where $S^{-} \pi^{-}, S^{-} \pi^{-\prime}$ are the predecessors of $S \pi, S \pi^{\prime}$ in $\mathcal{F}(\mathbf{s}), \mathcal{F}\left(\mathbf{s}^{\prime}\right)$ respectively.

Proposition 5. Under (L), (SN), (SS), (EG) and (IIP), we have, for any scenario s, any $S \pi \in \mathcal{F}(\mathbf{s})$ and its predecessor $S^{-} \pi^{-}$in $\mathcal{F}(\mathbf{s})$

$$
\psi_{i}^{\mathbf{s}}(v)=\gamma_{s, s^{-}}\left(v(S \pi)-v\left(S^{-} \pi^{-}\right)\right)
$$

for all $i \in S \backslash S^{-}$. If $S$ is a singleton, $\gamma_{s, s^{-}}$is denoted by $\gamma_{1,0}$.

Proof. Under (L), (SN), (SS), (EG), we already know that $\psi_{i}^{\mathbf{s}}(v)$ has the form (5), with coefficients $\gamma_{\tau(\mathbf{s}),|\pi|}$. Fix $S^{-} \subset S$, any $i \in S \backslash S^{-}$and any two scenarios $\mathbf{s}, \mathbf{s}^{\prime}$ defined as above. Then by (IIP) we get

$$
\gamma_{\tau(\mathbf{s}),|\pi|}=\gamma_{\tau\left(\mathbf{s}^{\prime}\right),\left|\pi^{\prime}\right|} \cdot
$$

Since $\pi$ and $\pi^{\prime}$ can be any partitions containing $S$, the coefficients do not depend on $\pi$. Now, $\tau(\mathbf{s})$ has the form $\ldots,\left(s^{-}, \rho\left(\pi^{-}\right),\left(i^{-}, j^{-}\right)\right), \ldots,(s, \rho(\pi),(i, j)), \ldots$, and any sequence of that type with $s, s^{-}$fixed is produced by some $\mathbf{s}$ defined as above. Consequently, the dependency to $\tau(\mathbf{s})$ is reduced to the dependency to $s, s^{-}$.

Theorem 2. The egalitarian scenario-value is the unique scenario-value satisfying (L), (SN), (SS), (EG), (E) and (IIP).

(see proof in the Appendix)

\subsection{Explicit expression of the coalition formation value}

Theorem 3. For any game $v$, the coalition formation value is given by

$$
\begin{aligned}
\phi_{i}(v) & =\frac{1}{n} v(N\{N\})+\sum_{\substack{T \sigma \in \Sigma(N) \backslash\{N\{N\}\} \\
T \ni i}} \frac{2(n-k) !}{n ! n !}(k-1)(k-1) !(k-2) ! t ! t_{2} ! \cdots t_{k} ! v(T \sigma) \\
& -\sum_{\substack{T \sigma \in \Sigma(N) \\
T \not i}} \frac{2 t(n-k) !}{n ! n !}(k-1) !(k-2) ! t !\left(t_{2}-1\right) ! \cdots t_{k} ! v(T \sigma),
\end{aligned}
$$

where $\sigma:=\left\{T, T_{2}, \ldots, T_{k}\right\}$, and it is assumed in the third term that $i \in T_{2}$, the second block of $\sigma$. 
(see proof in the Appendix) An equivalent expression, although less computationally efficient, is:

$$
\begin{aligned}
\phi_{i}(v) & =\frac{1}{n} v(N\{N\}) \\
& +\sum_{\substack{T \sigma \in \Sigma(N) \\
T \not \supset i T_{2} \supset\{i\}}} \frac{2 t(n-k) !}{n ! n !}(k-1) !(k-2) ! t !\left(t_{2}-1\right) ! \cdots t_{k} !\left[\frac{t+1}{t} v\left(T \cup i \sigma_{T \cup i}\right)-v(T \sigma)\right] \\
& -\sum_{\substack{T \sigma \in \Sigma(N) \\
T \not \supset i, T_{2}=\{i\}}} \frac{2 t(n-k) !}{n ! n !}(k-1) !(k-2) ! t ! t_{3} ! \cdots t_{k} ! v(T \sigma)
\end{aligned}
$$

with $\sigma_{T \cup i}$ the partition obtained from $\sigma$ by moving $i \in T_{2}$ to $T$.

Using (7), we obtain for a three players game $(i, j, k$ denote any three different players):

$$
\begin{aligned}
\phi_{i}(v) & =\frac{1}{3} v(N\{N\})+\frac{1}{9} v(i j\{i j, k\})+\frac{1}{9} v(i k\{i k, j\})-\frac{2}{9} v(j k\{i, j k\}) \\
& +\frac{1}{9} v(i\{i, j k\})-\frac{1}{18} v(j\{j, i k\})-\frac{1}{18} v(k\{k, i j\}) \\
& +\frac{2}{9} v(i\{i, j, k\})-\frac{1}{9} v(j\{i, j, k\})-\frac{1}{9} v(k\{i, j, k\}) .
\end{aligned}
$$

It can be checked that this differs from the other values proposed so far for PFF games (Myerson, Bolger, de Clippel and Serrano, Albizuri, etc., see Section 7).

\subsection{Relation with the Shapley value}

If we consider a PFF game with no externality, that is, $v(S \pi)=v\left(S \pi^{\prime}\right)$ for any $\pi$ and $\pi^{\prime}$ with $\pi \ni S, \pi^{\prime} \ni S$, it naturally corresponds to a TU game. We can define the TU game $\hat{v}$ from the PFF game $v$ by:

$$
\hat{v}(S):=v(S \pi) \text { for any } S \in 2^{N} \text {, any } \pi \ni S .
$$

Most of the values for PFF games coincide with the Shapley value for TU games when there is no externality. Applying (8) to a game with no externality we find

$$
\phi_{i}(\hat{v})=\frac{1}{3} \hat{v}(N)+\frac{1}{9} \hat{v}(i j)+\frac{1}{9} \hat{v}(i k)-\frac{2}{9} \hat{v}(j k)+\frac{1}{3} \hat{v}(i)-\frac{1}{6} \hat{v}(j)-\frac{1}{6} \hat{v}(k),
$$

which differs from the original Shapley value. We elaborate more on this surprising result, and claim that we are able to recover the classical Shapley 
value, provided we use the weighted version of our value. In addition, we will get an interesting interpretation of the Shapley value in terms of coalition formation. Let us consider as above a game without externalities. Recall that the Shapley value can be interpreted as the average marginal contribution of players when considering all possible ways for the players to enter one by one the game, i.e., all permutations on $N$. Now note that this can be represented as particular processes of coalition formation. Indeed, suppose that players enter the game in the order $1,2, \ldots, n$. This corresponds to the process

$$
\{1,2,3, \ldots, n\} \rightarrow\{12,3,4, \ldots, n\} \rightarrow\{123,4, \ldots, n\} \rightarrow \cdots \rightarrow\{123 \cdots n\},
$$

with the following convention: in each partition of the process, the first block is the block of players who have already entered the game (they already form a coalition), the other ones are the remaining players (they are still outside of the game). Note also that each process corresponds to two different orders: in the above example, $2,1, \ldots, n$ corresponds to the same process. Therefore, if we want to recover the Shapley value, only those processes should be taken (there are $2 / n$ ! such processes), i.e., their weight is $\frac{2}{n !}$, and the weight of the other ones is 0 . Now, consider the above process and the scenarios $\mathbf{s}_{1}$ of player 1 and $\mathbf{s}_{2}$ of player 2 :

$$
\begin{aligned}
& 1\{1,2,3, \ldots, n\} \rightarrow 12\{12,3,4, \ldots, n\} \rightarrow 123\{123,4, \ldots, n\} \rightarrow \cdots \rightarrow N\{N\} \\
& 2\{1,2,3, \ldots, n\} \rightarrow 12\{12,3,4, \ldots, n\} \rightarrow 123\{123,4, \ldots, n\} \rightarrow \cdots \rightarrow N\{N\} .
\end{aligned}
$$

Note that player 1 is the only one present from the beginning in the order $1,2, \ldots, n$, and similarly for player 2 in the order $2,1, \ldots, n$. Hence only these players can have an internal view of the process, i.e., only scenarios $\mathbf{s}_{1}, \mathbf{s}_{2}$ must be considered. We claim:

Proposition 6. The Shapley value for the TU game $\hat{v}$ is obtained by

$\phi_{i}^{\mathrm{S}}(\hat{v})=\frac{2}{n !} \sum_{\mathbf{p}_{\sigma}}\left(\frac{1}{2} \phi_{i}^{\mathbf{s}_{\sigma(1)} \leftarrow \mathbf{p}_{\sigma}}(v)+\frac{1}{2} \phi_{i}^{\mathbf{s}_{\sigma(2)} \leftarrow \mathbf{p}_{\sigma}}(v)\right)=\frac{1}{n !} \sum_{\mathbf{p}_{\sigma}}\left(\phi_{i}^{\mathbf{s}_{\sigma(1)} \leftarrow \mathbf{p}_{\sigma}}(v)+\phi_{i}^{\mathbf{s}_{\sigma(2)} \leftarrow \mathbf{p}_{\sigma}}(v)\right)$,

for $i \in N$, and $\mathbf{s}_{\sigma(1)} \leftarrow \mathbf{p}_{\sigma}$ denotes the scenario of player $\sigma(1)$ in the process of type (9) induced by $\sigma$, that is

$$
\{\sigma(1), \sigma(2), \sigma(3), \ldots, \sigma(n)\} \rightarrow\{\sigma(1) \sigma(2), \sigma(3), \sigma(4), \ldots, \sigma(n)\} \rightarrow \cdots \rightarrow\{N\}
$$

Proof. By definition of the Shapley value as an average of marginal contributions, we have

$$
\phi_{i}^{\mathrm{S}}(\hat{v})=\frac{1}{n !} \sum_{\sigma}\left(\hat{v}\left(S_{\sigma^{-1}(i)}^{\sigma}\right)-\hat{v}\left(S_{\sigma^{-1}(i)-1}^{\sigma}\right)\right)
$$


with $S_{i}^{\sigma}:=\{\sigma(1), \ldots, \sigma(i)\}$, and $S_{0}:=\emptyset$.

Consider the process $\mathbf{p}_{\sigma}$ induced by the permutation $\sigma$, and the two orders corresponding to $\mathbf{p}_{\sigma}$, namely $\sigma(1), \sigma(2), \ldots, \sigma(n)$ and $\sigma(2), \sigma(1), \ldots, \sigma(n)$. It suffices to show that for any player, the marginal contributions for these two orders in (10) coincide with the term for $\mathbf{p}_{\sigma}$.

Consider first any player $i \neq \sigma(1), \sigma(2)$. Then its marginal contribution is identical for these two orders, and is in total $\frac{2}{n !}\left(\hat{v}\left(S_{\sigma^{-1}(i)}^{\sigma}\right)-\hat{v}\left(S_{\sigma^{-1}(i)-1}^{\sigma}\right)\right.$ for the two orders. Consider now player $\sigma(1)$. In the first order, its contribution is $\frac{1}{n !} \hat{v}(\sigma(1))$, while it is $\frac{1}{n !}(v(\sigma(1) \sigma(2))-v(\sigma(2)))$ in the second order. The situation is symmetric for player $\sigma(2)$.

On the other hand, we have for any player $i \neq \sigma(1), \sigma(2)$ :

$$
\begin{aligned}
\phi_{i}^{\mathbf{s}_{\sigma(1)} \leftarrow \mathbf{p}_{\sigma}}(v) & =v\left(S_{\sigma^{-1}(i)}^{\sigma}\left\{S_{\sigma^{-1}(i)}^{\sigma}, \pi_{N \backslash S_{\sigma^{-1}(i)}^{\sigma}}^{\perp}\right\}\right)-v\left(S_{\sigma^{-1}(i)-1}^{\sigma}\left\{S_{\sigma^{-1}(i)-1}^{\sigma}, \pi_{N \backslash S_{\sigma^{-1}(i)-1}^{\sigma}}^{\perp}\right\}\right) \\
& =\hat{v}\left(S_{\sigma^{-1}(i)}^{\sigma}\right)-\hat{v}\left(S_{\sigma^{-1}(i)-1}^{\sigma}\right) \\
& =\phi_{i}^{\mathbf{s}_{\sigma(2)} \leftarrow \mathbf{p}_{\sigma}}(v),
\end{aligned}
$$

where we used the notation $\pi_{S}^{\perp}$ to denote a partition of a set $S$ in singletons. Similarly, we obtain for $i=\sigma(1), \sigma(2)$ :

$$
\begin{aligned}
& \phi_{\sigma(1)}^{\mathbf{s}_{\sigma(1)} \leftarrow \mathbf{p}_{\sigma}}(v)=\hat{v}(\sigma(1)) \\
& \phi_{\sigma(1)}^{\mathbf{s}_{\sigma(2)} \leftarrow \mathbf{p}_{\sigma}}(v)=\hat{v}(\sigma(1) \sigma(2))-\hat{v}(\sigma(2)),
\end{aligned}
$$

which proves the desired equality.

Hence, the difference between the classical Shapley value and our value lies in the discarded scenarios $\mathbf{s}_{3}, \ldots, \mathbf{s}_{n}$ in each process $\mathbf{p}_{\sigma}$ and the discarded processes. While it is clear why processes other than the $\mathbf{p}_{\sigma}$ 's should be discarded, let us explain why those scenarios are discarded. In Shapley's view, there is a distinction between players already in the game (those in the room) and those still outside the game. Note that in process (9), only player 1 or player 2 can be first in the room and be present during all the process of formation of the grand coalition, and these correspond precisely to scenarios $\mathbf{s}_{1}, \mathbf{s}_{2}$. By contrast, in a coalition formation process, all players are always present in the game, only the structure of the society evolves. Therefore, all scenarios have to be taken into account. In summary, a well-defined coalition formation value should never collapse to the classical Shapley value.

Similarly, it is possible to recover the value $\phi^{\mathrm{CS}}$ of de Clippel and Serrano [5] (and therefore of Pham Do and Norde [7], since they coincide, see 
Section 7). Its formula is

$$
\phi_{i}^{\mathrm{CS}}(v)=\phi_{i}^{\mathrm{S}}(\tilde{v}), \quad i \in N,
$$

where $\tilde{v}(S):=v\left(S\left\{S, \pi_{N \backslash S}^{\perp}\right\}\right)$. Due to the definition of $\tilde{v}$, we see that the proof of Proposition 6 can be applied without any change. Therefore

$$
\phi_{i}^{\mathrm{CS}}(v)=\frac{1}{n !} \sum_{\mathbf{p}_{\sigma}}\left(\phi_{i}^{\mathbf{s}_{\sigma(1)} \leftarrow \mathbf{p}_{\sigma}}(v)+\phi_{i}^{\mathbf{s}_{\sigma(2)} \leftarrow \mathbf{p}_{\sigma}}(v)\right), \quad i \in N .
$$

\section{Application to the Cournot oligopoly ex- ample}

We come back to our motivating example of Section 3, and solve it with our proposed value. Since in this example, we are interested into specific processes, the adequate notion is the process-value.

We begin by considering a 4-person game with $N=\{1,2,3,4\}$, and the two typical coalition formation processes $\mathbf{p}$ and $\mathbf{p}^{\prime}$, defined in Section 3:

$$
\begin{array}{r}
\mathbf{p}:\{1,2,3,4\} \rightarrow\{12,3,4\} \rightarrow\{123,4\} \rightarrow\{1234\} \\
\mathbf{p}^{\prime}:\{1,2,3,4\} \rightarrow\{12,3,4\} \rightarrow\{12,34\} \rightarrow\{1234\}
\end{array}
$$

The Shapley process-values of the processes $\mathbf{p}$ and $\mathbf{p}^{\prime}$ are given by

$$
\begin{aligned}
\phi^{\mathbf{p}} & =\left(\frac{2 v_{1}+v_{2}+3 v_{3}}{24}, \frac{2 v_{1}+v_{2}+3 v_{3}}{24}, \frac{v_{1}+5 v_{2}-3 v_{3}}{12}, \frac{3 v_{1}-2 v_{2}}{4}\right) \\
\phi^{\mathbf{p}^{\prime}} & =\left(\frac{v_{1}}{4}, \frac{v_{1}}{4}, \frac{v_{1}}{4}, \frac{v_{1}}{4}\right)
\end{aligned}
$$

These are the averages of the four Shapley scenario-values induced from the corresponding processes $\mathbf{p}$ and $\mathbf{p}^{\prime}$. These values are interpreted as average contributions of the players in the processes. They are symmetric for $\mathbf{p}^{\prime}$, but not for $\mathbf{p}$, as expected. Note that $\phi^{\mathbf{p}}$ does not depend on $v_{4}$ and $\phi_{1}^{\mathbf{p}}=\phi_{2}^{\mathbf{p}}$. This is because in the first step of coalition formation process $\mathbf{p}$, the roles of players 1 and 2 are very similar since both are first players to enter, and $v_{4}$ has no influence in that case. Here we could compare the contributions of the same firm in the two different processes.

This result can be extended to the general case. First we give the Shapley process-value for the following coalition formation process $\mathbf{p}$ in an $n$-person game.

$$
\mathbf{p}:\{1,2,3, \ldots, n\} \rightarrow\{12,3, \ldots, n\} \rightarrow\{123, \ldots, n\} \rightarrow \cdots \rightarrow\{N\}
$$


The value becomes

$$
\phi_{k}^{\mathbf{p}}=\frac{1}{n}\left[v_{n-k+2}+(k-1)\left(v_{n-k+1}-v_{n-k+2}\right)+\sum_{j=k}^{n-1} \frac{1}{j}\left(v_{n-j}-v_{n-j+1}\right)\right]
$$

for $k=2, \ldots, n-1$, and

$$
\phi_{1}^{\mathbf{p}}=\phi_{2}^{\mathbf{p}}, \quad \phi_{n}^{\mathbf{p}}=\frac{1}{n}\left[v_{2}+(n-1)\left(v_{1}-v_{2}\right)\right] .
$$

Consider next the case of $n=2^{m}$ and a process $\mathbf{p}^{\prime}$ defined by

$\mathbf{p}^{\prime}:\{1,2,3,4, \ldots, n\} \rightarrow\{12,3,4, \ldots, n\} \rightarrow\{12,34, \ldots, n\} \rightarrow \cdots \rightarrow\{12,34, \ldots,(n-1) n\}$

$\rightarrow\{1234, \ldots,(n-1) n\} \rightarrow \cdots \rightarrow\{1234, \ldots,(n-3)(n-2)(n-1) n\} \rightarrow \cdots$

$\rightarrow\{12345678, \ldots,(n-3)(n-2)(n-1) n\} \rightarrow \cdots \rightarrow\left\{123 \cdots\left(2^{m-1}\right),\left(2^{m-1}+1\right) \cdots n\right\} \rightarrow\{N\}$

The Shapley process-value for process $\mathbf{p}^{\prime}$ is given by $\phi_{k}^{\mathbf{p}^{\prime}}=\frac{v_{1}}{n}$ for $k=1, \ldots, n$, which shows that everyone has a perfectly symmetric role in this process.

Next we apply this to the Cournot oligopoly problem, with $v_{k}=\frac{1}{(k+1)^{2}}$ (see Section 3). We find

$$
\begin{gathered}
\phi_{k}^{\mathbf{p}}=\frac{1}{n(n-k+3)^{2}}+\frac{(k-1)(2 n-2 k+5)}{n(n-k+2)^{2}(n-k+3)^{2}}+\sum_{j=k}^{n-1} \frac{2 n-2 j+3}{n j(n-j+1)^{2}(n-j+2)^{2}} \\
\quad \text { for } k=2, \ldots, n-1, \\
\phi_{1}^{\mathbf{p}}=\phi_{2}^{\mathcal{P}}, \quad \phi_{n}^{\mathbf{p}}=\frac{5 n-1}{36 n} \\
\phi_{k}^{\mathbf{p}^{\prime}}=\frac{1}{4 n} \quad \text { for } k=1,2, \ldots, n,
\end{gathered}
$$

Moreover it holds that for $k=2, \ldots, n-1$,

$\phi_{k+1}^{\mathbf{p}}-\phi_{k}^{\mathbf{p}}=\frac{1}{n}\left[v_{n-k+1}-v_{n-k+2}+\left(k-\frac{1}{k}\right)\left(v_{n-k}-v_{n-k+1}\right)-(k-1)\left(v_{n-k+1}-v_{n-k+2}\right)\right]$.

Since $v_{k}=\frac{1}{(k+1)^{2}}$ is a concave decreasing function, the Shapley process-value $\phi_{k}^{\mathbf{p}}$ of player $k$ satisfies $\phi_{1}^{\mathbf{p}}=\phi_{2}^{\mathbf{p}}<\phi_{3}^{\mathbf{p}} \ldots<\phi_{n}^{\mathbf{p}}$, because $v_{n-k+1}-v_{n-k+2}>$ $0, k-\frac{1}{k}>k-1$ and $v_{n-k}-v_{n-k+1}>v_{n-k+1}-v_{n-k+2}$.

This shows that when players enter the game one by one, the later a player joins the current coalition, the more the contribution of this player in Cournot oligopoly, as expected. 
For the sake of completeness, we give numerical results for the 4-person case.

$$
\phi^{\mathbf{p}} \approx \frac{1}{4}(.138, .138, .206, .528), \quad \phi^{\mathbf{p}^{\prime}}=\frac{1}{4}(.25, .25, .25, .25) .
$$

The last player gets more profit in process $\mathbf{p}$, which is more than one half.

Symmetric values for PFF games give the equal division $\frac{1}{4}(.25, .25, .25, .25)$ and do not say anything about a coalition formation process. However our Shapley process-value shows the difference between two processes $\mathbf{p}$ and $\mathbf{p}^{\prime}$. Process $\mathbf{p}^{\prime}$ is more egalitarian than process $\mathbf{p}$. Even in a symmetric oligopoly, the coalition formation process matters a lot.

\section{Related works and concluding remarks.}

As we discussed in the three person example (Section 5.4), the coalition formation value differs from other values of PFF games, since our value reflects the whole (dynamic) process of formation of coalitions, while the others one are more related to the coalitional (static) view of games. Here we make a comparison of the different axiom systems.

Myerson's value of a PFF game (Myerson [14]) is uniquely determined by the following three axioms: (S: symmetry) for any permutation $\sigma, \psi_{i}(v)=$ $\psi_{\sigma i}(\sigma v)$, (ADD: additivity) for any two games $v, w, \psi(v+w)=\psi(v)+\psi(w)$, and (CAR: carrier) if $T$ is a carrier, that is, $v(T \pi)=v(T \cap S, \pi \wedge\{T, N \backslash$ $T\}) \forall S \pi \in \Sigma(N)$, then $\sum_{k \in T} \psi_{k}(v)=v(N,\{N\})$. (ADD) is a bit weaker but almost the same as (L). (CAR) is an extension of that in TU games, which is equivalent to the null axiom and the efficiency for the original Shapley value. In this sense it is a direct generalization of an axiom system for the original Shapley value. The most different axiom from our axiomatization of our scenario-value is (CAR). It is important to mention that the Myerson's value of a PFF game in three person game is not monotonic: if $v(i\{i, j, k\})$ increases, the Myerson's value of player $i$ decreases.

Bolger's value (Bolger [4]) of a PFF game is uniquely determined by the following five axioms: (S),(L), (E: efficiency), (B-null) if $j$ is a null player, that is, $v(S \pi)=v\left(S \backslash\{j\} \pi^{\prime}\right)$ for any $S, \pi, \pi^{\prime}$ such that $j \in S \in \pi, \pi^{\prime}=$ $\left\{T \cup j, S_{1} \backslash j, \ldots, S_{k} \backslash j\right\}$ with $\pi=\left\{T, S_{1}, \ldots, S_{k}\right\}, j \in N \backslash T$, then $\psi_{j}(v)=0$, and (MM: Modified Marginality) for any two games $v, w$, and $i \in N$, if for any $S \ni i$, and $\pi \ni S, \sum_{T \in \pi, T \neq S}\left[v(S, \pi)-v\left(S \backslash i, \alpha_{i T}\right)\right]=\sum_{T \in \pi, T \neq S}[w(S, \pi)-$ $\left.w\left(S \backslash i, \alpha_{i T}\right)\right]$, then $\psi_{i}(v)=\psi_{i}(w)$, where for $i \in S, \pi=\left\{S, T, S_{1}, S_{2}, \ldots, S_{k}\right\}$, $\alpha_{i T}=\left\{S \backslash\{i\}, T \cup i, S_{1}, \ldots, S_{k}\right\}$. Axioms (MM) as well as (B-null) are needed, which are both very strong. Again (B-null) is very different from our $(\mathrm{SN})$. 
Pham Do and Norde ([7]) proposed a value of a PFF game which is uniquely determined by the following four axioms: (S), (ADD), (E) and (DNnull): if $j$ is a null player, that is, $v(S, \pi \cup\{\{j\}\})=v(S \cup\{j\},(\pi \backslash\{S\}) \cup\{S \cup$ $j\}$ ) for any $S \subseteq N \backslash\{j\}$, for any $\pi \in \Pi(N \backslash\{j\})$, then $\phi_{j}(v)=0$. Fujinaka [8] gives a different axiomatization of the same value using (M:Marginality) for any two games $v, w$, if $v(S \cup\{j\},(\pi \backslash\{S\}) \cup\{S \cup j\})-v(S, \pi \cup\{\{j\}\})=$ $w(S \cup\{j\},(\pi \backslash\{S\}) \cup\{S \cup j\})-w(S, \pi \cup\{\{j\}\})$ for any $S \subseteq N \backslash\{j\}$, for any $\pi \in \Pi(N \backslash\{j\})$, then $\phi_{j}(v)=\phi_{j}(w)$. He characterizes it by (S), (ADD), and (M). de Clippel and Serrano ([5]) also give another axiomatization of the same value using a balanced contribution axiom. Again the key difference is based on the null axiom (DN-null). The important remark is that the formula of their value of a PFF game $v$ is given by $\phi(v)=$ Shapley $(\tilde{v})$, where $\tilde{v}(S)=v\left(S,\left\{S,\{k\}_{k \in N \backslash S}\right\}\right)$, and Shapley $(\tilde{v})$ is the Shapley value of TU game $\tilde{v}$. This means their value does not utilize the full information of the PFF game.

Macho-Stadler et al. ([13]) proposed a collection of values for PFF games, called average values. They are characterized by (SS: strong symmetry), which is a stronger version of symmetry, (L), (E), and (B-null). The typical representative of this family is given by this formula: $\phi(v)=\operatorname{Shapley}(\bar{v})$, where $\bar{v}(S)=\frac{1}{\#\{\pi \in \Pi \mid \pi \ni S\}} \sum_{\{\pi \in \Pi \mid \pi \ni S\}} v(S \pi)$. It satisfies in addition (SI: similar influence): if players $i$ and $j$ have similar influence in games $v, v^{\prime}$, that is, $v=v^{\prime}$ except for 2 elements $S\left\{S, i, j, S_{2}, \ldots, S_{k}\right\}$ and $S\left\{S,\{i, j\}, S_{2}, \ldots, S_{k}\right\}$, and $v\left(S\left\{S, i, j, S_{2}, \ldots, S_{k}\right\}\right)=v^{\prime}\left(S\left\{S,\{i, j\}, S_{2}, \ldots, S_{k}\right\}\right), v\left(S\left\{S,\{i, j\}, S_{2}, \ldots, S_{k}\right\}\right)=$ $v^{\prime}\left(S\left\{S, i, j, S_{2}, \ldots, S_{k}\right\}\right)$, then $\psi_{i}(v)=\psi_{i}(w)$, and $\psi_{j}(v)=\psi_{j}(w)$. This value is also characterized by Albizuri et al. [1]. Clearly it utilizes the full information given by the PFF game.

Our scenario-value is different from any of the values above, mainly because it is not the (classical) Shapley value of some TU game induced by the PFF game. Therefore, the null player axiom of the Shapley value of TU games naturally induces the above special null axioms for PFF games, which all differ from our null axiom. Moreover, we claim that the underlying structure of embedded coalitions (which is not explicitely mentionned in the above cited works) is implicitly suggested by the null axioms which are employed. Indeed, in the case of TU games, the null axiom is based on the difference between the worths of $S$ and $S \backslash i$, assuming $S \ni i$. These elements are "neighbors" in the lattice $\left(2^{N}, \subseteq\right)$. In the PFF case, our scenario null axiom is defined along a maximal chain of $\Sigma(N)_{\perp}$. The B-null axiom takes the difference of worth between $S\left\{S, S_{2}, \ldots, S_{k}\right\}$ and $S \backslash i\left\{S \backslash i, S_{2} \cup i, \ldots, S_{k}\right\}$, for $S \ni i$. In $\Sigma(N)_{\perp}$, these elements are not neighbors because they are on the same level. To recover them as neighbors, one possibility is the following: 
take the Boolean lattice $\left(2^{N}, \subseteq\right)$. Duplicate each element $S$ as many times there are different possible coalition structures containing $S$, and indicate these coalition structures. Put all possible links between duplicates of an element $S$ and duplicates of an element $T$ if and only if these elements are linked in the Boolean lattice. Doing so, the B-nullity condition appears for neighbors elements. This structure also explains well the average approach: it can almost be seen on the picture. For illustration, we give $\Sigma(N)$ and the structure induced by B-nullity for $n=3$ on Figures 3 and 4 .

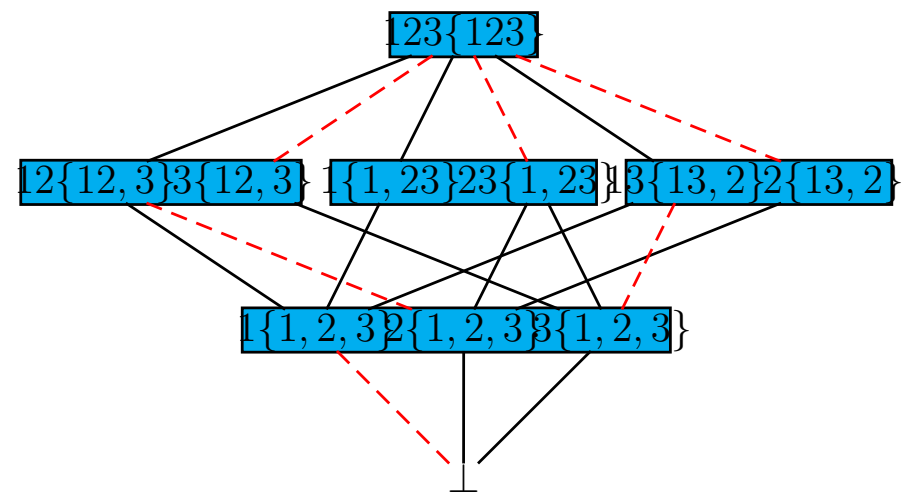

Figure 3: Diagram of $\left(\Sigma(3)_{\perp}, \leq\right)$. Elements with the same partition are framed in grey. Elements in nullity condition of player 1 are linked by a dashed line

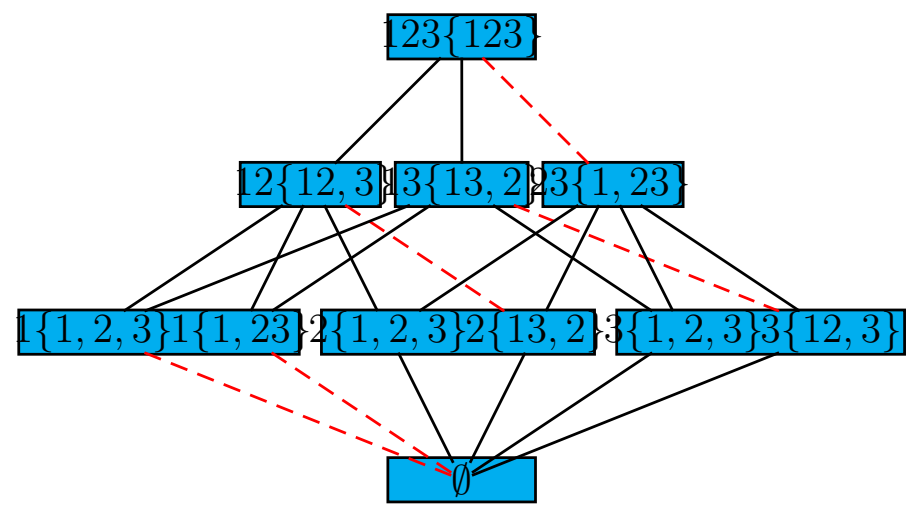

Figure 4: Diagram of the structure induced by the B-null axiom, used by Bolger, and Macho-Stadler et al. $n=3$. Elements with same coalition (duplicates) are framed in grey. Elements in B-nullity condition of player 1 are linked by a dashed line 


\section{References}

[1] M. Albizuri, J. Arin, and J. Rubio. An axiom system for a value for games in partition function form. Int. Game Theory Review, 7:63-72, 2005 .

[2] G. E. Andrews. The theory of partitions, volume 2 of Encyclopedia of mathematics and its applications. Addison-Wesley, 1976.

[3] F. Bloch. Sequential formation of coalitions in games with externalities and fixed payoff divisions. Games and Economic Behavior, 14:90-123, 1996.

[4] E. M. Bolger. A set of axioms for a value for partition function games. Int. J. of Game Theory, 18:37-44, 1989.

[5] G. de Clippel and R. Serrano. Marginal contributions and externalities in the value. Econometrica, 76(6):1413-1436, 2008.

[6] E. Diamantoudi and L. Xue. Coalitions, agreements and efficiency. J. of Economic Theory, 136:105-125, 2007.

[7] K. H. Pham Do and H. Norde. The Shapley value for partition function form games. International Game Theory Review, 9:353-360, 2007.

[8] Y. Fujinaka. On the marginality principle in partition function form games. Technical report, Kobe University, 2004.

[9] Y. Funaki and T. Yamato. The core of an economy with a common pool resource: A partition function approach. International Journal of Game Theory, 28:157-171, 1999.

[10] Y. Funaki and T. Yamato. Stable coalition structures under restricted coalitional changes. GLOPE working paper, Waseda University, 2010.

[11] I. Gilboa and E. Lehrer. Global games. Int. J. of Game Theory, 20:129147, 1991.

[12] M. Grabisch. The lattice of embedded subsets. Discrete Applied Mathematics, 158:479-488, 2010. doi: 10.1016/j.dam.2009.10.015.

[13] I. Macho-Stadler, D. Pérez-Castillo, and D. Wettstein. Sharing the surplus: An extension of the Shapley value for environments with externalities. J. of Economic Theory, 135:339-356, 2007. 
[14] R. B. Myerson. Values of games in partition function form. International Journal of Game Theory, 6:23-31, 1977.

[15] D. Ray and R. Vohra. Equilibrium binding agreement. J. of Economic Theory, 73:30-78, 1997.

[16] R. M. Thrall and W. F. Lucas. n-person games in partition function form. Naval Research Logistic Quarterly, 10:281-298, 1963.

\section{Appendix - Proofs of theorems}

\section{Proof of Theorem 1}

The fact that the egalitarian scenario-value satisfies (L), (SN), (SS), (EG) and (SE) is easy to check and left to the reader.

Conversely, let $\mathbf{s}=\left\{\emptyset \pi^{\perp}, S_{1} \pi_{1,1}, \ldots, S_{1} \pi_{1, m_{1}}, S_{2} \pi_{2,1}, \ldots, S_{2} \pi_{2, m_{2}}, \ldots, S_{k} \pi_{k, 1}, \ldots\right.$, $\left.S_{k} \pi_{k, m_{k}}, N\{N\}\right\}$ be fixed.

From Proposition 4 we get:

$$
\begin{aligned}
\sum_{i \in N} \psi_{i}^{\mathbf{s}}(v)= & \gamma_{\tau(\mathbf{s}),\left|\pi_{1, m_{1}}\right|} v\left(S_{1} \pi_{1, m_{1}}\right)+\left|S_{2} \backslash S_{1}\right| \gamma_{\tau(\mathbf{s}),\left|\pi_{2, m_{2}}\right|}\left(v\left(S_{2} \pi_{2, m_{2}}\right)-v\left(S_{1} \pi_{1, m_{1}}\right)\right)+ \\
& \left.\cdots+\left|S_{j} \backslash S_{j-1}\right| \gamma_{\tau(\mathbf{s}), \mid \pi_{j, m_{j}}} \mid v\left(S_{j} \pi_{j, m_{j}}\right)-v\left(S_{j-1} \pi_{j-1, m_{j-1}}\right)\right)+ \\
& \cdots+\left|N \backslash S_{k}\right| \gamma_{\tau(\mathbf{s}), 1}\left(v(N\{N\})-v\left(S_{k} \pi_{k, m_{k}}\right)\right) \\
= & \left(\gamma_{\tau(\mathbf{s}),\left|\pi_{1, m_{1}}\right|}|-| S_{2} \backslash S_{1}\left|\gamma_{\tau(\mathbf{s}),\left|\pi_{2, m_{2}}\right|}\right| v\left(S_{1} \pi_{1, m_{1}}\right)+\right. \\
& \cdots+\left(\left|S_{j} \backslash S_{j-1}\right| \gamma_{\tau(\mathbf{s}),\left|\pi_{j, m_{j}}\right|}-\left|S_{j+1} \backslash S_{j}\right| \gamma_{\tau(\mathbf{s}),\left|\pi_{j+1, m_{j+1}}\right|}\right) v\left(S_{j} \pi_{j, m_{j}}\right)+ \\
& \cdots+\left|N \backslash S_{k}\right| \gamma_{\tau(\mathbf{s}), 1} v(N\{N\}) .
\end{aligned}
$$

From the (SE) axiom, we deduce the following linear system of $k+1$ equations and $k+1$ unknowns:

$$
\begin{aligned}
\gamma_{\tau(\mathbf{s}),\left|\pi_{1, m_{1}}\right|}-\left|S_{2} \backslash S_{1}\right| \gamma_{\tau(\mathbf{s}),\left|\pi_{2, m_{2}}\right|} & =0 \\
\vdots & =0 \\
\left|S_{j} \backslash S_{j-1}\right| \gamma_{\tau(\mathbf{s}),\left|\pi_{j, m_{j}}\right|}-\left|S_{j+1} \backslash S_{j}\right| \gamma_{\tau(\mathbf{s}),\left|\pi_{j+1, m_{j+1}}\right|} & =0 \\
\vdots & =0 \\
\left|N \backslash S_{k}\right| \gamma_{\tau(\mathbf{s}), 1} & =1 .
\end{aligned}
$$

Evidently the system is nonsingular, since from the last equation $\gamma_{\tau(\mathbf{s}), 1}$ is obtained, then substituting it into the last but one, we get $\gamma_{\tau(\mathbf{s}),\left|\pi_{k, m_{k}}\right|}$ and so on. Knowing that the coefficients of the egalitarian scenario-value are solutions of the system, it is the unique solution. 


\section{Proof of Theorem 2}

Again, the egalitarian-scenario-value clearly satisfies these axioms.

Conversely, let us assume that the scenario-value satisfies the five axioms, and let us compute $\sum_{i \in N} \sum_{\mathbf{s} \in \mathcal{S}} \psi_{i}^{\mathbf{s}}(v)$. From $\sum_{i \in N} \sum_{\mathbf{s} \in \mathcal{S}} \psi_{i}^{\mathbf{s}}(v)=v(N\{N\})$, we get a system of linear equations, one per element $S \pi$. We know that there exists at least one solution to this system, since our egalitarian scenario-value satisfies the five axioms. Our task will be to prove that this is the only solution. To this aim, we will prove that there are at least as many equations as variables, and that there exists a subsystem which can be made triangular.

First, we determine the form of the equation for element $S \pi:=S\left\{S, S_{2}, \ldots, S_{k}\right\}$, assuming $S \pi$ is any element different from $N\{N\}$ and $S$ is not a singleton. From (6), there is a negative contribution for $v(S \pi)$ with coefficient $-\gamma_{s^{+}, s}$ for all $S^{+} \pi^{+}$such that $S^{+} \pi^{+} \sqsupset S \pi,\left|S^{+}\right|=s^{+}, \pi^{+}:=\left\{S^{+}, S_{2}^{+}, \ldots, S_{k^{+}}^{+}\right\}$, and $S^{+}=S \cup S_{j}$, for some $j=2, \ldots, k$, for all $i \in S^{+} \backslash S$, and all scenarios s passing through $S^{+} \pi^{+}$and $S \pi$, such that $S^{+} \pi^{+}, S \pi \in \mathcal{F}(\mathbf{s})$. Hence, any scenario of the following form, with $S^{+}=S \cup S_{j}$ :

$\perp, \ldots, S \pi, \underbrace{S \cup S_{j}}_{S^{+}} \pi_{S \cup S_{j}}, \ldots, \underbrace{S \cup S_{j}}_{S^{+}} \pi^{+}, S^{+} \cup S_{l}^{+} \pi_{S^{+} \cup S_{l}^{+}}^{+}, \ldots, N\{N\}, \quad l=2, \ldots, k^{+}$

will lead to a negative contribution with coefficient $-\gamma_{s^{+}, s}$. The notation $\pi_{S \cup S_{j}}$, etc., is a shorthand for $\left(\pi \backslash\left\{S, S_{j}\right\}\right) \cup\left\{S \cup S_{j}\right\}$. The set of all such scenarios (for $j$ fixed, i.e., $S^{+}$fixed) is obtained as all possible combinations of:

(i) all sub-scenarios from $\perp$ to $S \pi$;

(ii) all sub-scenarios from $S \cup S_{j} \pi_{S \cup S_{j}}$ to $S \cup S_{j} \pi^{+}$, for all possible $\pi^{+}$ coarser than $\pi_{S \cup S_{j}}$ and containing $S \cup S_{j}$;

(iii) for a given $\pi^{+}$, all sub-scenarios from $S^{+} \cup S_{l}^{+} \pi_{S^{+} \cup S_{l}^{+}}^{+}$to the top $N\{N\}$, for $l=2, \ldots, k^{+}$.

Hence the number of such scenarios is

$$
\beta_{s+s_{j}, s, \pi}=|\mathcal{C}([\perp, S \pi])| \sum_{\substack{\pi^{+}>\pi \\ \pi^{+} \ni S \cup S_{j}}}\left(\left|\mathcal{C}\left(\left[\pi_{S \cup S_{j}}, \pi^{+}\right]\right)\right| \times \sum_{l=2}^{k^{+}}\left|\mathcal{C}\left(\left[S^{+} \cup S_{l}^{+} \pi_{S^{+} \cup S_{l}^{+}}^{+}, N\{N\}\right]\right)\right|\right),
$$

where the notation $\mathcal{C}([\perp, S \pi])$ stands for the set of maximal chains from $\perp$ to $S \pi$, and so on (for the second term, since the coalition is always $S^{+}$, we can omit it). Although this number seems difficult to compute (!), it depends ultimately only on $k, s, s_{2}, \ldots s_{k}$, hence on the number of blocks of $\pi$ and their cardinality. Indeed, the following results are shown in [12]. 
- Consider two distinct elements $\pi, \pi^{\prime} \in \Pi(n)$, with $\pi^{\prime}<\pi$. Then

$$
\left|\mathcal{C}\left(\left[\pi^{\prime}, \pi\right]\right)\right|=\frac{\left(k^{\prime}-k\right) !}{2^{k^{\prime}-k}} l_{1} ! l_{2} ! \cdots l_{k} !
$$

with $\pi:=\left\{S_{1}, \ldots, S_{k}\right\}, \pi^{\prime}:=\left\{S_{11}, \ldots, S_{1 l_{1}}, S_{21}, \ldots, S_{2 l_{2}}, \ldots, S_{k l_{k}}\right\}$, with $\left\{S_{i 1}, \ldots, S_{i l_{i}}\right\}$ a partition of $S_{i}, i=1, \ldots, k$, and $k^{\prime}:=\sum_{i=1}^{k} l_{i}$.

- Let $S \pi$ be an embedded coalition, with $\pi:=\left\{S, S_{2} \ldots, S_{k}\right\}$. The number of maximal chains from $\perp$ to $S \pi$ is

$$
\mid \mathcal{C}([\perp, S \pi)]) \mid=\frac{s(n-k) !}{2^{n-k}} s ! s_{2} ! \cdots s_{k} !
$$

- Let $S \pi$ be an embedded coalition, with $\pi:=\left\{S, S_{2} \ldots, S_{k}\right\}$. The number of maximal chains from $S \pi$ to $N\{N\}$ is

$$
|\mathcal{C}([S \pi, N\{N\}])|=\frac{1}{k}\left|\mathcal{C}\left(\Sigma(k)_{\perp}\right)\right|=\frac{k !(k-1) !}{2^{k-1}} .
$$

We deduce from this:

$$
\begin{aligned}
\left|\mathcal{C}\left(\left[\pi_{S \cup S_{j}}, \pi^{+}\right]\right)\right| & =\frac{\left(k-1-k^{+}\right) !}{2^{k-1-k^{+}} 1 . l_{2}^{+} ! \cdots l_{k^{+}}^{+} !} \\
\left|\mathcal{C}\left(\left[S^{+} \cup S_{l}^{+} \pi_{S^{+} \cup S_{l}^{+}}^{+}, N\{N\}\right]\right)\right| & =\frac{\left(k^{+}-1\right) !\left(k^{+}-2\right) !}{2^{k^{+}-2}}
\end{aligned}
$$

with $l_{2}^{+}, \ldots, l_{k^{+}}^{+}$being the numbers of blocks in $\pi_{S \cup S_{j}}$ corresponding to $S_{2}^{+}, \ldots, S_{k+}^{+}$. Clearly, $\mid \mathcal{C}([\perp, S \pi)]) \mid$ depends only on $k$ and the cardinality of blocks of $\pi$. For $\left|\mathcal{C}\left(\left[\pi_{S \cup S_{j}}, \pi^{+}\right]\right)\right|$, doing the summation over $\pi^{+}$, we have that $k^{+}$will vary from 2 to $k-1$. Accordingly, each $l_{j}^{+}$will vary from 1 (when $k^{+}=k-1$ ) to $k$ (when $k^{+}=2$ ). Hence the second term (after summation) depends only on $k$. Similarly, $\left|\mathcal{C}\left(\left[S^{+} \cup S_{l}^{+} \pi_{S^{+} \cup S_{l}^{+}}^{+}, N\{N\}\right]\right)\right|$ depends only on $k$.

Similarly, there is a positive contribution for $v(S \pi)$ with coefficient $\gamma_{s, s^{-}}$ for all $S^{-} \pi^{-}$such that $S^{-} \pi^{-} \sqsubset S \pi,\left|S^{-}\right|=s^{-}$, and $S=S^{-} \cup S_{1}^{-}$for some $S_{1}^{-} \in \pi^{-}$, all $i \in S \backslash S^{-}$, and all scenarios $\mathbf{s}$ passing through elements $S \pi$ and $S^{-} \pi^{-}$, so that $S \pi, S^{-} \pi^{-} \in \mathcal{F}(\mathbf{s})$. Hence, any scenario of the following form, with $S^{-} \pi^{-}$defined as above

$\perp, \ldots, S^{-} \pi^{-}, \underbrace{S^{-} \cup S_{1}^{-}}_{S} \pi_{S^{-} \cup S_{1}^{-}}^{-}, \ldots, S \pi, S \cup S_{j} \pi_{S \cup S_{j}}, \ldots, N\{N\}, \quad j=2, \ldots, k$ 
will lead to a positive contribution with coefficient $\gamma_{s, s^{-}}$. The number of such scenarios is, reasoning as above,

$$
\alpha_{s, s^{-}, \pi}=\sum_{\substack{S^{-} \subset S,\left|S^{-}\right|=s^{-} \\ \pi^{-}<\pi, \pi^{-} \ni S^{-}, S_{1}^{-} \\ \text {s.t. } S=S^{-} \cup S_{1}^{-}}}\left(\left|\mathcal{C}\left(\left[\perp, S^{-} \pi^{-}\right]\right)\right| \times\left|\mathcal{C}\left(\left[\pi_{S^{-} \cup S_{1}^{-}}^{-}, \pi\right]\right)\right|\right) \times \sum_{j=2}^{k}\left|\mathcal{C}\left(\left[S \cup S_{j} \pi_{S \cup S_{j}}, N\{N\}\right]\right)\right|
$$

Again, this number depends only on $s^{-}$, the number of blocks of $\pi$ and their cardinality. In summary, the equation for $S \pi \neq N\{N\}$ is

$$
\sum_{s^{-}<s}\left(s-s^{-}\right) \alpha_{s, s^{-}, \pi} \gamma_{s, s^{-}}-\sum_{j=2}^{k} s_{j} \beta_{s+s_{j}, s, \pi} \gamma_{s+s_{j}, s}=0 .
$$

Let us address briefly the case of singletons and $N$. If $S=\{i\}$, the first term is replaced by $\alpha_{1,0, \pi} \gamma_{1,0}$, with

$$
\alpha_{1,0, \pi}=\sum_{j=2}^{k}\left|\mathcal{C}\left(\left[S \cup S_{j} \pi_{S \cup S_{j}}, N\{N\}\right]\right)\right| .
$$

If $S=N$, the second term does not exist. In summary:

$$
\begin{array}{rlrl}
\alpha_{1,0, \pi} \gamma_{1,0}+\sum_{j=2}^{k}\left(s^{+}-s\right) \beta_{s+s_{j}, s, \pi} \gamma_{s+s_{j}, s} & =0, & & (S \text { is a singleton }) \\
\sum_{n^{-}<n}\left(n-n^{-}\right) \alpha_{n, n^{-},\{N\}} \gamma_{n, n^{-}} & =v(N\{N\}), & (S \pi=N\{N\}) .
\end{array}
$$

From the above considerations, equations for $S \pi$ and $S^{\prime} \pi^{\prime}$ will be identical if and only if $s=s^{\prime}$, and $\pi$ and $\pi^{\prime}$ are of the same type (same number of blocks and same cardinality of blocks). Hence, the number of different equations for $|S|=s$ is the number of integer partitions of $n-s$, denoted by $p(n-s)$, for $s=1, \ldots, n$ (for $n=s$, there is only one equation, which is (11). Hence we put $p(0):=1)$. For example, the numbers of integer partitions of $1,2,3$, 4 are respectively $1,2,3,5$. Hence, for $n=2,3,4,5$ we have respectively 2 , 4,7 , and 12 different equations.

The number of variables $\gamma_{s, s^{\prime}}$ is much easier to compute. For $s=1$, there is only one variable, namely $\gamma_{1,0}$. For $1<s \leq n$ fixed, $s^{\prime}$ varies from 1 to $s-1$. Hence the total number of variables is:

$$
1+\sum_{s=2}^{n}(s-1)=\sum_{s=2}^{n} s-n+2=\frac{n^{2}-n+2}{2} .
$$


This gives for $n=2,3,4,5$ players, $2,4,7,11$ variables. It is easy to see that this number is less or equal than the number of equations. Indeed, for large $n$, the following formula is known:

$$
p(n) \approx \frac{1}{4 n \sqrt{3}} \exp \left(\pi\left(\frac{2 n}{3}\right)^{\frac{1}{2}}\right)
$$

which is clearly exponential (see Andrews [2]).

It remains to find a subsystem of equations which can be made triangular. Let us order the variables as follows: $\gamma_{1,0}, \gamma_{2,1}, \gamma_{3,1}, \gamma_{3,2}, \gamma_{4,1}, \ldots$ For each variable $\gamma_{s^{\prime}, s}$, except for $\gamma_{1,0}$, let us find an equation using only variables up to $\gamma_{s^{\prime}, s}$. It suffices to take the equation for $S \pi$ such that the largest block of $\pi \backslash\{S\}$ is of size $s^{\prime}-s$. Doing so for all $\gamma_{s^{\prime}, s}$, we form a subsystem. If in this subsystem, we put for each equation $\gamma_{1,0}$ on the right side, the subsystem becomes triangular. So it has a unique solution in terms of $\gamma_{1,0}$, which can be determined by substituting all variables in the equation corresponding to $S \pi=N\{N\}$. This proves the uniqueness of the solution.

\section{Proof of Theorem 3}

We consider the elementary games $e_{T \sigma}$ for any $T \sigma \in \Sigma(N)$, defined by $e_{T \sigma}\left(T^{\prime} \sigma^{\prime}\right)=1$ iff $T \sigma=T^{\prime} \sigma^{\prime}$, and 0 otherwise. We have for any game $v$ in $\Gamma(N), v=\sum_{T \sigma \in \Sigma(N)} v(T \sigma) e_{T \sigma}$. Since the egalitarian scenario-value is linear, so is the value. Hence, for any game $v$ in $\Gamma(N)$,

$$
\phi_{i}(v)=\sum_{T \sigma \in \Sigma(N)} v(T \sigma) \phi_{i}\left(e_{T \sigma}\right)
$$

It remains to compute $\phi_{i}\left(e_{T \sigma}\right)$ for all $i \in N$ and all $T \sigma \in \Sigma(N)$.

A first important thing is to notice that symmetry (SS) implies $\phi_{i}\left(e_{T \sigma}\right)=$ $\phi_{j}\left(e_{T \sigma}\right)$ for all $i, j \in T$. Indeed, consider any permutation $\tau$ such that $\tau(T)=$ $T$, and $\tau$ is the identity on $N \backslash T$. Then, for any $i \in T$,

$\phi_{i}\left(e_{T \sigma}\right)=\sum_{\mathbf{s}} \phi_{i}^{\mathbf{s}}\left(e_{T \sigma}\right)=\sum_{\mathbf{s}} \phi_{\tau(i)}^{\tau(\mathbf{s})}\left(e_{T \sigma} \circ \tau^{-1}\right)=\sum_{\mathbf{s}^{\prime}} \phi_{\tau(i)}^{\mathbf{s}^{\prime}}\left(e_{\tau(T \sigma)}\right)=\sum_{\mathbf{s}^{\prime}} \phi_{\tau(i)}^{\mathbf{s}^{\prime}}\left(e_{T \sigma}\right)=\phi_{\tau(i)}\left(e_{T \sigma}\right)$.

From this and efficiency we immediately have:

$$
\phi_{i}\left(e_{N\{N\}}\right)=\frac{1}{n}, \quad \forall i \in N .
$$

Let us consider a given $T \sigma \in \Sigma(N), T \sigma \neq N\{N\}$, and consider any $i \notin T$. Let us put for convenience $\sigma=\left\{T, T_{2}, \ldots, T_{k}\right\}$ and consider that $i \in T_{2}$. A 
scenario $\mathbf{s}$ induces $\phi_{i}^{\mathbf{s}}\left(e_{T \sigma}\right) \neq 0$ if and only if $\mathbf{s}$ contains $T \sigma$ and the successor of $T \sigma$ in $\mathbf{s}$ is $T \cup T_{2} \sigma_{T \cup T_{2}}$, with $\sigma_{T \cup T_{2}}:=\left(\sigma \backslash\left\{T, T_{2}\right\}\right) \cup\left\{T \cup T_{2}\right\}$, and in this case $\phi_{i}^{\mathbf{s}}\left(e_{T \sigma}\right)=-\frac{1}{t_{2}}$. The number of such scenarios is (see proof of Th. 2)

$$
\frac{t(n-k) !}{2^{n-2}} t ! t_{2} ! \cdots t_{k} !(k-1) !(k-2) !
$$

hence

$$
\phi_{i}\left(e_{T \sigma}\right)=-\frac{2 t(n-k) !}{n ! n !}(k-1) !(k-2) ! t !\left(t_{2}-1\right) ! \cdots t_{k} !, \quad \forall i \in T_{2} .
$$

Establishing the corresponding formulas when $i \in T_{3}, \ldots, T_{k}$ successively we deduce

$$
\sum_{i \notin T} \phi_{i}\left(e_{T \sigma}\right)=-\frac{2 t(k-1)(n-k) !}{n ! n !} t ! t_{2} ! \cdots t_{k} !(k-1) !(k-2) !,
$$

hence, by efficiency we get:

$$
\begin{aligned}
\sum_{i \in T} \phi_{i}\left(e_{T \sigma}\right) & =0-\sum_{i \notin T} \phi_{i}\left(e_{T \sigma}\right) \\
& =\frac{2 t(k-1)(n-k) !}{n ! n !} t ! t_{2} ! \cdots t_{k} !(k-1) !(k-2) !
\end{aligned}
$$

which gives by symmetry:

$$
\phi_{i}\left(e_{T \sigma}\right)=\frac{2(n-k) !}{n ! n !}(k-1)(k-1) !(k-2) ! t ! t_{2} ! \cdots t_{k} !, \quad \forall i \in T .
$$

Hence for any game $v$, we obtain:

$$
\begin{aligned}
\phi_{i}(v) & =\frac{1}{n} v(N\{N\})+\sum_{\substack{T \sigma \in \Sigma(N) \backslash\{N\{N\}\} \\
T \ni i}} \frac{2(n-k) !}{n ! n !}(k-1)(k-1) !(k-2) ! t ! t_{2} ! \cdots t_{k} ! v(T \sigma) \\
& -\sum_{\substack{T \sigma \in \Sigma(N) \\
T \ngtr i}} \frac{2 t(n-k) !}{n ! n !}(k-1) !(k-2) ! t !\left(t_{2}-1\right) ! \cdots t_{k} ! v(T \sigma) .
\end{aligned}
$$

